\title{
Rational surfaces with linear normals and their convolutions with rational surfaces
}

\author{
Maria Lucia Sampoli ${ }^{\mathrm{a}, *}$, Martin Peternell ${ }^{\mathrm{b}}$, Bert Jüttler ${ }^{\mathrm{c}}$ \\ a Università di Siena, Dipartimento di Scienze Matematiche ed Informatiche, Italy \\ ${ }^{\mathrm{b}}$ Technische Universität Wien, Inst. für Diskrete Mathematik und Geometrie, Austria \\ c J. Kepler Universität Linz, Institut für Angewandte Geometrie, Austria
}

Received 21 February 2005; received in revised form 28 June 2005; accepted 6 July 2005

Available online 15 August 2005

\begin{abstract}
It is shown that polynomial (or rational) parametric surfaces with a linear field of normal vectors are dual to graphs bivariate polynomials (or rational functions). We discuss the geometric properties of these surfaces. In particular, using the dual representation it is shown that the convolution with general rational surfaces yields again rational surfaces. Similar results hold in the case of curves.
\end{abstract}

○ 2005 Elsevier B.V. All rights reserved.

Keywords: Rational curves and surfaces; Linear normals; Dual representation; Convolution; Offsetting

\section{Introduction}

The notions of convolution surfaces and Minkowski sums in two and three dimensions are used in various fields of geometric applications, e.g., mathematical morphology, computer graphics, convex geometry and computational geometry, and there is a close connection between the two concepts of convolution surfaces and Minkowski sums. Given two sets $\mathcal{A}$ and $\mathcal{B}$ in $\mathbb{R}^{d}$, their Minkowski sum is the set formed by the sums of all pairs of vectors in the Cartesian product $\mathcal{A} \times \mathcal{B}$. On the other hand, the convolution surface of the two surfaces consists of the sums of all pairs of vectors with parallel surface

\footnotetext{
* Corresponding author.

E-mail address: sampoli@unisi.it (M.L. Sampoli).
} 


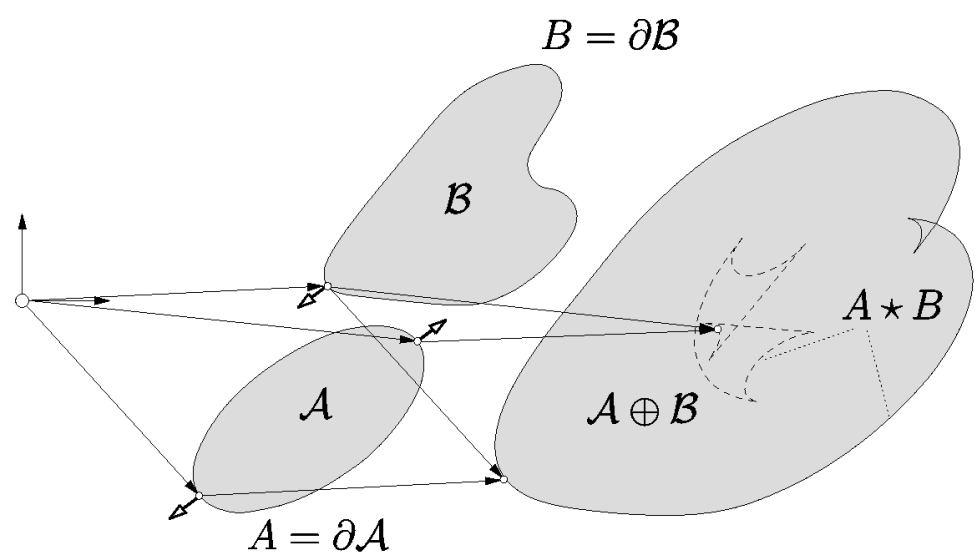

(a)

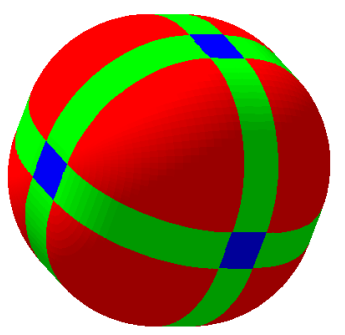

(b)

Fig. 1. (a) Minkowski sum of two planar domains and the convolution of their boundaries. (b) Minkowski sum of a ball and a cube.

normals. The boundary surface of the Minkowski sum is contained in the convolution surface of the two original boundary surfaces, provided that they have a well-defined normal everywhere.

Fig. 1(a) illustrates these notions in the planar case. The convolution $A \star B$ of the two boundaries $A=\partial \mathcal{A}$ and $B=\partial \mathcal{B}$ contains the boundary of the Minkowski sum $\mathcal{A} \oplus \mathcal{B}$. It consists of two components, generated by points with parallel normals having the same (solid curve) and the opposite (dashed curve) orientation, respectively. Fig. 1(b) shows the Minkowski sum of a ball and a cube. The boundary consists of segments of spheres and cylinders and planar patches.

In the curve case, various algorithms for computing Minkowski sums exist (Kaul and Farouki, 1995; Kohler and Spreng, 1995; Lee et al., 1998a, 1998b; Ramkumar, 1996; Farouki, 2003). The main issue is to trim away those parts of the convolution curve that do not contribute to the outer boundary of the Minkowski sum. Another important problem consists in finding an exact description of the convolution. Though the set of algebraic curves and surfaces is closed under convolution, this result is of little practical value, since the resulting degrees are far too high to be useful. Also, one is often interested in curves and surfaces that admit a rational parametric representation, since they can easily be fed into standard CAD systems.

As an important special case, offset surfaces (convolutions with spheres) have thoroughly been discussed, where certain rational surfaces are equipped with rational offset surfaces. For instance, this is true for surfaces which degenerate to space curves, and for quadrics (Landsmann et al., 2001; Lü, 1994; Peternell and Pottmann, 1998; Schicho, 2000; Schicho, 1998; Sendra and Sendra, 2000).

Rational convolution surfaces of more general surfaces did not receive much attention so far. A first approach was studied recently by Seong et al. (2002), while Mühlthaler and Pottmann (2003) have analyzed the case of two ruled surfaces. Convolutions between paraboloids and general rational surfaces were analyzed by Peternell and Manhart (2003).

In this paper, we generalize the latter result to the case of convolutions between surfaces with linear normals (LN surfaces) and general rational surfaces. LN surfaces, which were studied in (Jüttler and Sampoli, 2000) have sufficient flexibility to model smooth surfaces without parabolic points. Convolution of LN surfaces have been studied by Sampoli (2005). Here, we will show that the convolution of LN 
surfaces with general parametric surfaces have explicit parametric representation, which are even rational for rational surfaces.

This paper is organized as follows. The first three sections are devoted to LN surfaces, their dual representation, and the available constructions. Then we discuss the so-called relative differential geometry of these surfaces. Sections 6 and 7 discuss Minkowski sums, convolution surfaces, and the parameterization of convolution surfaces. Finally, we conclude this paper.

\section{Preliminaries}

This section gives an introduction to LN surfaces and discusses some important geometric properties.

Definition 1. Consider a polynomial (or, more general, a rational) surface $\mathbf{p}(u, v)$. This surface is said to be an $\mathbf{L N}$ surface, if its normal vectors admit a linear representation of the form

$$
\overrightarrow{\mathbf{N}}(u, v)=\overrightarrow{\mathbf{a}} u+\overrightarrow{\mathbf{b}} v+\overrightarrow{\mathbf{c}}
$$

with certain constant coefficient vectors $\overrightarrow{\mathbf{a}}, \overrightarrow{\mathbf{b}}, \overrightarrow{\mathbf{c}} \in \mathbb{R}^{3}$. More precisely, it satisfies the equations

$$
\mathbf{p}_{u}(u, v) \cdot \overrightarrow{\mathbf{N}}(u, v) \equiv \mathbf{p}_{v}(u, v) \cdot \overrightarrow{\mathbf{N}}(u, v) \equiv 0,
$$

where $\mathbf{p}_{u}(u, v)=(\partial / \partial u) \mathbf{p}(u, v), \mathbf{p}_{v}(u, v)=(\partial / \partial v) \mathbf{p}(u, v)$.

Eqs. (2) can be seen as linear constraints on the space of polynomial or rational parametric surfaces, and this approach has been used by Jüttler and Sampoli (2000) for generating LN surface patches matching given Hermite boundary data. In this paper, we will study the geometrical properties by using the so-called dual representation of these surfaces, where the surface is seen as the envelope of its tangent planes.

Remark 2. (1) If the three vectors $\overrightarrow{\mathbf{a}}, \overrightarrow{\mathbf{b}}, \overrightarrow{\mathbf{c}}$ are linearly dependent, then the surface $\mathbf{p}(u, v)$ describes a general cylinder, since the unit normals $\overrightarrow{\mathbf{N}} /\|\overrightarrow{\mathbf{N}}\|$ are contained in a great circle on the unit sphere. In particular if all three vectors are proportional, the surface is simply a plane.

(2) In the remainder of this paper we assume that the three vectors are linearly independent. Without loss of generality we may then assume that

$$
\overrightarrow{\mathbf{a}}=(1,0,0)^{\top}, \quad \overrightarrow{\mathbf{b}}=(0,1,0)^{\top}, \quad \overrightarrow{\mathbf{c}}=(0,0,1)^{\top},
$$

i.e., $\overrightarrow{\mathbf{N}}(u, v)=(u, v, 1)^{\top}$. This situation can be achieved as follows. Firstly, we scale the normals such that the plane (1) spanned by them has distance 1 from the origin of the coordinate system. Secondly, we rotate the LN surface around the origin, such that the plane spanned by the normals becomes the plane $z=1$. Finally, we apply a linear parameter transformation $u=u\left(u^{\prime}, v^{\prime}\right), v=v\left(u^{\prime}, v^{\prime}\right)$ to the surface, in order to get a normal field (1) satisfying (3).

Proposition 3. Under the assumptions of Remark 2(2), the tangent planes of an LN surface have the equations

$$
T(u, v): \quad f(u, v)+u x+v y+z=0,
$$


where $f(u, v)=-\mathbf{p}(u, v) \cdot \overrightarrow{\mathbf{N}}(u, v)$ is a polynomial or rational function, in the case of a polynomial or rational LN surface, respectively. On the other hand, given a system of tangent planes of the form (4) with a polynomial or rational function $f(u, v)$, the envelope surface

$$
\mathbf{p}(u, v)=\left(-f_{u},-f_{v},-f+u f_{u}+v f_{v}\right)^{\top}
$$

is a polynomial or rational LN surface.

Proof. The envelope surface $\mathbf{p}=(x, y, z)$ satisfies the equations

$$
\begin{array}{ll}
T(u, v): & f(u, v)+u x+v y+z=0, \\
T_{u}(u, v): & f_{u}(u, v)+x=0, \\
T_{v}(u, v): & f_{v}(u, v)+y=0,
\end{array}
$$

and the normal vector evaluates to

$$
\overrightarrow{\mathbf{N}}(u, v)=\left(f_{u u} f_{v v}-f_{u v}^{2}\right)(u, v, 1)^{\top} .
$$

Remark 4. Due to (7), singular points of the envelope surface (5) are characterized by $f_{u u} f_{v v}-f_{u v}^{2}=0$. In addition, the Gaussian curvature of the envelope equals

$$
K(u, v)=\frac{1}{\left(f_{u u} f_{v v}-f_{u v}^{2}\right)\left(1+u^{2}+v^{2}\right)^{2}},
$$

since $K=\operatorname{det}(H) / \operatorname{det}(G)$ is the quotient of the determinants of the fundamental forms of the surface. These determinants evaluate to

$$
\operatorname{det}(G)=\left(1+u^{2}+v^{2}\right)\left(f_{u u} f_{v v}-f_{u v}^{2}\right)^{2}, \quad \operatorname{det}(H)=\frac{f_{u u} f_{v v}-f_{u v}^{2}}{1+u^{2}+v^{2}} .
$$

If the envelope surface possesses both hyperbolic $(K<0)$ and elliptic $(K>0)$ points, the corresponding domains are separated by the singular curve $C$, which is determined by the algebraic curve $f_{u u} f_{v v}-f_{u v}^{2}=$ 0 in the $(u, v)$-parameter domain.

\section{The dual representation}

There exist several interesting relations between the LN-surfaces $\mathbf{p}(u, v)$ defined by a polynomial or rational function $f$ and the associated graph surface

$$
\mathbf{q}(u, v)=(u, v, f(u, v))^{\top},
$$

since the graph surface is dual to the LN surface in the sense of projective geometry.

The points of $\mathbf{q}(u, v)$ are elliptic, parabolic or hyperbolic, if the sign of

$$
\operatorname{det} H(f)=f_{u u} f_{v v}-f_{u v}^{2}
$$

is 1,0 or -1 , respectively. Clearly, the parabolic points either form an algebraic curve, or the entire graph surface consists of parabolic points only. In the latter case, $\mathbf{q}(u, v)$ is a general cylinder surface.

Corollary 5. Elliptic and hyperbolic points of the graph surface $\mathbf{q}(u, v)$ correspond to elliptic and hyperbolic points of the $L N$ surface $\mathbf{p}(u, v)$. Parabolic points of the graph surface $\mathbf{q}(u, v)$ correspond to singular points of $\mathbf{p}(u, v)$. 
Proof. These facts are consequences of (7), (8) and (10).

Remark 6. Graph surfaces $\mathbf{q}(u, v)$, which are general cylinders, correspond to singular surfaces $\mathbf{p}(u, v)$, which degenerate into planar curves. More precisely, the function $f$ can be assumed to take the form

$$
f(u, v)=d u+g(v)
$$

with a real constant $d$ and a rational function $g(v)$. The envelope surface (5) degenerates into the planar curve

$$
\left(-d,-g^{\prime}(v),-g(v)+v g^{\prime}(v)\right)^{\top} .
$$

If the envelope surface has a self-intersection (i.e., a double line), then its points correspond to pairs of points of $\mathbf{q}(u, v)$ with coinciding tangent planes. Consequently, if $f$ is a convex function, then the envelope does not have any self-intersections.

We illustrate these observations by a first example of an LN surface, see Fig. 2. The function $f$ is equal to $u^{3}-v^{3}$, and the LN surface has the parametric representation $\mathbf{p}(u, v)=\left(-3 u^{2}, 3 v^{2}, 2 u^{3}-2 v^{3}\right)^{\top}$. The parabolic lines (marked with $\mathrm{P}$ ) on the graph surface are $u=0$ and $v=0$. The associated LN surface has 2 edges of regression $(E)$, which intersect in the point $(0,0,0)$. Each of them is a planar cubic curve with a cusp (i.e., equivalent to Neil's parabola). In addition, it has a double line, which corresponds to the double tangent planes along the curve $u=v$, since the tangent planes at $(u, u)$ and $(-u,-u)$ are identical.
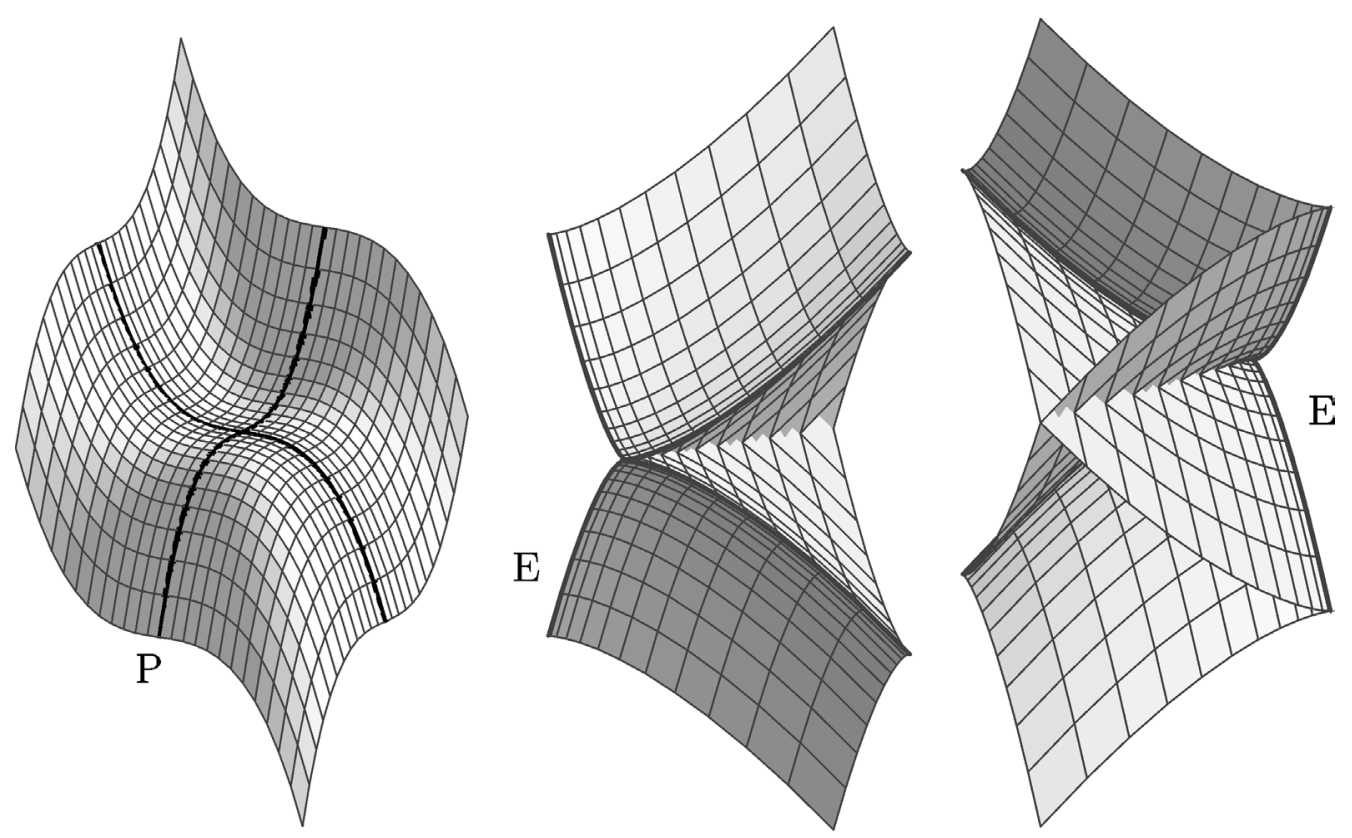

Fig. 2. Graph surface (left) of a cubic polynomial and the associated LN surface (center and right). 


\section{Construction of $\mathrm{LN}$ surfaces}

We summarize two constructions of LN surfaces via Hermite interpolation. For both of them, the input consists of three points $\mathbf{v}_{i} \in \mathbb{R}^{3}$ with associated normal vectors $\overrightarrow{\mathbf{n}}_{i} \in \mathbb{R}^{3}(i=0,1,2)$. The normal vectors are not assumed to be normalized.

\subsection{The problem}

Let us consider the problem of finding an LN surface patch that interpolates three given points (vertices) $\mathbf{v}_{i} \in \mathbb{R}^{3}$ along with the associated normal vectors $\overrightarrow{\mathbf{n}}_{i} \in \mathbb{R}^{3}$. Both constructions generate a triangular surface patch $\mathbf{p}(u, v)$, whose parameter domain is a triangle $\triangle \subset \mathbb{R}^{2}$. The parameter pairs $(u, v) \in \triangle$ are described by their barycentric coordinates $(r, s, t)$ with respect to the domain triangle $\triangle$, i.e.,

$$
(u, v)=r \mathbf{w}_{1}+s \mathbf{w}_{2}+t \mathbf{w}_{3}, \quad \text { satisfying } \quad r+s+t=1
$$

where $\mathbf{w}_{1}, \mathbf{w}_{2}, \mathbf{w}_{3} \in \mathbb{R}^{2}$ are the vertices of $\triangle$.

The patch $\mathbf{p}(u, v)$ is either a triangular Bézier patch (cf. (Farin et al., 2002)) or a collection of such patches, which interpolates the given three points, i.e.,

$$
\mathbf{p}\left(\mathbf{w}_{i}\right)=\mathbf{v}_{i} .
$$

In addition, in order to produce a patch of an $\mathrm{LN}$ surface, the normal at a point $\mathbf{p}(u, v)$ is to be parallel to

$$
\overrightarrow{\mathbf{N}}(r, s, t)=r \mathbf{n}_{1}+s \mathbf{n}_{2}+t \mathbf{n}_{3},
$$

where $(r, s, t)$ are the barycentric coordinates of $(u, v)$, cf. (13). This implies the conditions

$$
\left.\frac{\partial}{\partial u} \mathbf{p}\right|_{(u, v)=r \mathbf{w}_{1}+s \mathbf{w}_{2}+t \mathbf{w}_{3}} \cdot \overrightarrow{\mathbf{N}}(r, s, t)=\left.\frac{\partial}{\partial v} \mathbf{p}\right|_{(u, v)=r \mathbf{w}_{1}+s \mathbf{w}_{2}+t \mathbf{w}_{3}} \cdot \overrightarrow{\mathbf{N}}(r, s, t)=0 .
$$

\subsection{Two constructions}

Both constructions consists of two steps.

(1) Construction of boundary curves. For any pair of points $\mathbf{v}_{i}, \mathbf{v}_{j}, i<j$, we construct a polynomial boundary curve $\mathbf{x}_{i, j}(t), t \in[0,1]$, of the triangular surface patch. In order to obtain patches which can be joined to form a globally $G^{1}$ surface, the boundaries should be fully determined by the points $\mathbf{v}_{i}, \mathbf{v}_{j}$ and vertex normals $\overrightarrow{\mathbf{n}}_{i}, \overrightarrow{\mathbf{n}}_{j}$.

The boundary $\mathbf{x}_{i, j}$ is to satisfy

$$
\mathbf{x}_{i, j}(0)=\mathbf{v}_{i}, \quad \mathbf{x}_{i, j}(1)=\mathbf{v}_{j}, \quad \mathbf{x}_{i, j}^{\prime}(t) \cdot\left[(1-t) \overrightarrow{\mathbf{n}}_{i}+t \overrightarrow{\mathbf{n}}_{j}\right] \equiv 0,
$$

where $\mathbf{x}^{\prime}=(\mathrm{d} / \mathrm{d} t) \mathbf{x}$. These conditions lead to linear equations for the coefficients of the polynomial curve, which are solvable, provided that the degree is sufficiently high. The remaining degrees of freedom are used to minimize a suitable energy functional, such as $\int_{0}^{1}\left(\mathbf{x}_{i j}^{\prime \prime}\right)^{2} \mathrm{~d} t$.

(2) Filling in a patch. In the second step, we generate a triangular surface patch whose boundary curves are given by $\mathbf{x}_{i, j}(t)$, and satisfy (16); this leads to a system of linear equations.

It turns out that it is generally not possible to fill in a single patch, due to compatibility conditions at the vertices (similar to the vertex enclosure problem). Two solutions to this problem are proposed: 


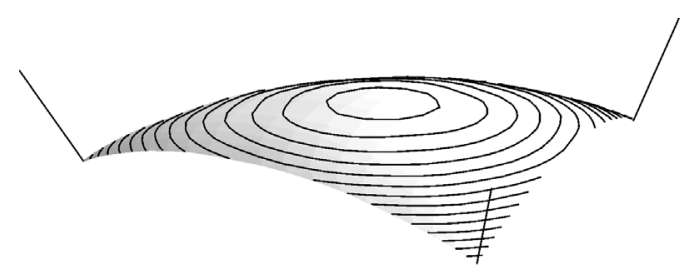

(a)

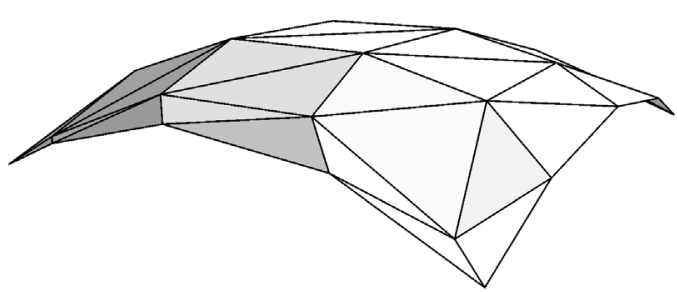

(c)

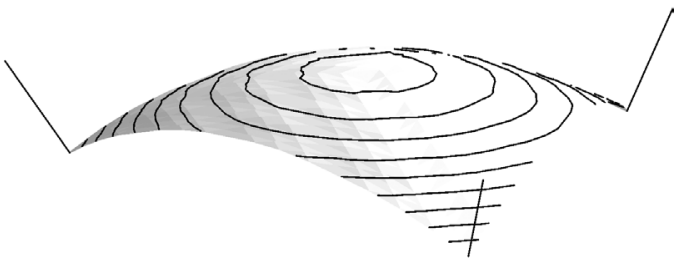

(b)

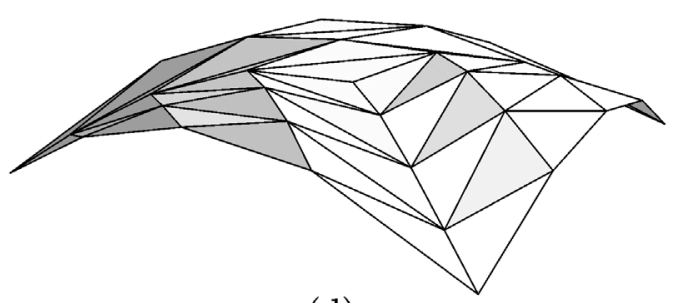

(d)

Fig. 3. LN surfaces interpolating three points with associated normal vectors (a), (b) and their control nets (c), (d). The surfaces have been generated using singularly parameterized surfaces (a), (c) and Clough-Tocher splits (b), (d).

(a) One may use a single patch with singular points at the vertices. This has to be taken into account already during the construction of the boundaries, which should then satisfy

$$
\mathbf{x}_{i, j}^{\prime}(0)=\mathbf{x}_{i, j}^{\prime}(1)=\overrightarrow{\mathbf{0}}
$$

in addition to (17). This approach leads to patches of degree 6. See (Jüttler, 1998) for details.

(b) Alternatively, in order to avoid potential problems with singular points, one may apply the Clough-Tocher split, by filling in a surface patch composed of three triangular surface patches. This leads to three patches of degree 4. This technique is described in (Jüttler and Sampoli, 2000).

Both approaches lead to systems of linear equations, and the remaining degrees of freedom can be used to minimize suitable fairness measures.

Two examples are shown in Fig. 3. Note that both constructions may produce surfaces which have sharp edges (singular curves), since the prescribed normal field limits the shape of the surface. According to our experience, the surface behaves nicely for boundary data which have been taken from an existing surface without parabolic points, provided that the distances between the sampled points are sufficiently small. This could even be proved for the boundary curves generated in the second construction (Jüttler and Sampoli, 2000).

\section{Relations between $\mathrm{LN}$-surfaces and the unit paraboloid}

In this section we point to some properties of LN-surfaces in connection with paraboloids. It will turn out that $\mathrm{LN}$-surfaces are in some sense generalizations of paraboloids. This property applies also to the computation of convolution surfaces in Section 7. 
We recall the parameterization $\mathbf{p}(u, v)=\left(-f_{u},-f_{v},-f+u f_{u}+v f_{v}\right)^{\top}$ of an LN-surface $\Phi$ and that its normal vectors are given by $\overrightarrow{\mathbf{N}}(u, v)=(u, v, 1)^{\top}$. Additionally we consider the paraboloid $Y$, represented by

$$
\mathbf{y}(u, v)=\left(u, v, \frac{1}{2}\left(1-u^{2}-v^{2}\right)\right)^{\top}=(u, v, y(u, v))^{\top} .
$$

Unlike Eq. (9), which is a dual representation, describing a set of tangent planes, the paraboloid $Y$ is given as a set of points ("primal" representation).

Up to a normalization, $Y$ 's normal vectors

$$
\overrightarrow{\mathbf{N}}_{y}(u, v)=\frac{1}{\sqrt{1+u^{2}+v^{2}}}(u, v, 1)^{\top}
$$

agree with those of $\Phi$. Obviously this implies that $\Phi$ 's tangent planes are parallel to those of $Y$.

Two points $\mathbf{p}$ of $\Phi$ and $\mathbf{y}$ of $Y$ are called corresponding, if their normal vectors $\overrightarrow{\mathbf{N}}$ and $\overrightarrow{\mathbf{N}}_{y}$ are parallel. Thus, this correspondence is realized by equal (surface) parameters $u, v$. In Section 7 , this correspondence applies to the construction of convolution surfaces.

Euclidean differential geometry investigates the unit normal vectors of a surface considered as parameterization of the unit sphere $S^{2}$. The shape operator or Weingarten mapping $w: \mathbf{p}_{u} \mapsto-\overrightarrow{\mathbf{N}}_{u}, \mathbf{p}_{v} \mapsto-\overrightarrow{\mathbf{N}}_{v}$ is the differential of the mapping $\mathbf{p}(u, v) \rightarrow-\overrightarrow{\mathbf{N}}$. At each point $\mathbf{p}(u, v), w$ is a linear mapping in the tangent plane. The eigenvalues and eigenvectors of $w$ are the principal curvatures and principal curvature directions of $\Phi$ at $\mathbf{p}$. by

By substituting $S^{2}$ by the 'unit' paraboloid $Y$, or in other words, according to the normalization of $\overrightarrow{\mathbf{N}}$

$$
\widetilde{\overrightarrow{\mathbf{N}}}(u, v)=(u, v, y(u, v))=\mathbf{y}(u, v)=\left(u, v, \frac{1}{2}\left(1-u^{2}-v^{2}\right)\right),
$$

$\widetilde{\overrightarrow{\mathbf{N}}}$ is considered as relative normalization with respect to $Y$. Expressing the second fundamental form of $\Phi$ with respect to $\widetilde{\overrightarrow{\mathbf{N}}}$, one obtains

$$
\widetilde{H}^{-1}=\left[\begin{array}{ll}
f_{u u} & f_{u v} \\
f_{u v} & f_{v v}
\end{array}\right], \quad \text { and } \quad \tilde{H}=\frac{1}{f_{u u} f_{v v}-f_{u v}^{2}}\left[\begin{array}{cc}
f_{v v} & -f_{u v} \\
-f_{u v} & f_{u u}
\end{array}\right] .
$$

$\widetilde{H}$ is the coordinate matrix of the relative shape operator

$$
\begin{aligned}
\tilde{w}: \mathbf{p}_{u} & =\left(-f_{u u},-f_{u v}, u f_{u u}+v f_{u v}\right) \mapsto-\widetilde{\overrightarrow{\mathbf{N}}}_{u}=-(1,0,-u), \\
\mathbf{p}_{v} & =\left(-f_{u v},-f_{v v}, u f_{u v}+v f_{v v}\right) \mapsto-\widetilde{\widetilde{\mathbf{N}}}_{v}=-(0,1,-v),
\end{aligned}
$$

with respect to $\widetilde{\overrightarrow{\mathbf{N}}}$. Analogously to the Euclidean case, the eigenvalues and eigenvectors of $\widetilde{H}$ are principal curvatures and principal curvature directions, with respect to the operator $\tilde{w}$. Since $Y$ is strongly convex, the eigenvalues and eigenvectors are always real. The concept of relative curvature theory is applied to computing generalized offset surfaces in (Pottmann, 1997). More information on this topic can be found e.g. in (Li et al., 1993). 


\section{Convolution surfaces and Minkowski sums}

Given two sets $\mathcal{A}$ and $\mathcal{B}$ in $\mathbb{R}^{d}$, the Minkowski sum of these sets is defined as

$\mathcal{A} \oplus \mathcal{B}=\{\mathbf{a}+\mathbf{b}, \mathbf{a} \in \mathcal{A}$ and $\mathbf{b} \in \mathcal{B}\}$,

see Fig. 1 for examples. In particular, algorithms for computing the Minkowski sum of closed (convex) polygons in the plane and polyhedral objects in space have been studied in computational geometry, see (Bajaj and Kim, 1989; Ramkumar, 1996; Kohler and Spreng, 1995). Applications include motion planning for polygonal objects in the presence of polygonal obstacles.

Later, these concepts have been generalized to arbitrary shapes in the plane and in space, see (Lee et al., 1998a, 1998b; Kaul and Farouki, 1995; Mühlthaler and Pottmann, 2003; Peternell and Manhart, 2003), where the notion of the convolution of two (not necessarily convex) objects has been introduced. ${ }^{1}$

Now we consider two regular surfaces $A$ and $B$ in three-dimensional space, which are given by parametric representations $\mathbf{a}(u, v)$ and $\mathbf{b}(s, t)$ with parameter domains $(u, v) \in \Omega_{A} \subseteq \mathbb{R}^{2}$ and $(s, t) \in \Omega_{B} \subseteq$ $\mathbb{R}^{2}$, respectively.

Definition 7. The convolution surface of two surfaces $A$ and $B$ is the set of points

$$
A \star B=\{\mathbf{a}+\mathbf{b} \mid \mathbf{a} \in A, \mathbf{b} \in B \text { and } \overrightarrow{\mathbf{M}}(\mathbf{a}) \| \overrightarrow{\mathbf{N}}(\mathbf{b})\},
$$

where $\overrightarrow{\mathbf{M}}(\mathbf{a})$ and $\overrightarrow{\mathbf{N}}(\mathbf{b})$ are the normal vectors of $A$ and $B$ at the points $\mathbf{a} \in A$ and $\mathbf{b} \in B$, and $\|$ denotes parallelism.

Remark 8. If one is interested in the boundaries of Minkowski sums, one may modify the definition by requiring - in addition to property of parallel normal vectors - that the vectors have the same orientation, $\overrightarrow{\mathbf{M}}(\mathbf{a})=\lambda(\mathbf{a}) \overrightarrow{\mathbf{N}}(\mathbf{b})$, with some positive factor $\lambda(\mathbf{a})$. On the other hand, the original version of the definition has the advantage of acting within the set of algebraic surfaces, since the convolution of two algebraic surfaces is again an algebraic surface. For instance, the two-sided offsets of conic sections are algebraic curves, while the one-sided offsets are generally not.

The sum of the coordinate vectors is computed only for those points whose normal vectors are parallel. The definition requires differentiability and regularity of the input surfaces $A$ and $B$, since otherwise normal vectors do not exist. A more general definition-which is beyond the scope of this paper-could be given by considering 'completed' normal fields.

While Definition 7 uses normal vectors, the convolution surface $A \star B$ is invariant under affine transformations of the objects $A$ and $B$. This is due to the fact that affine mappings preserve the parallelism of the tangent planes.

Note that there is a close relationship between convolution surfaces and Minkowski sums: the boundary of the Minkowski sum of two sets $\mathcal{A}, \mathcal{B}$ is contained in the convolution surface of the two boundary surfaces,

$$
\partial(\mathcal{A} \oplus \mathcal{B}) \subseteq(\partial \mathcal{A}) \star(\partial \mathcal{B}) .
$$

1 This notion should not be confused with the convolution of two functions $f$ and $g$, which represents roughly spoken, the overlap of $f$ and a reversed and translated version of $g$. 


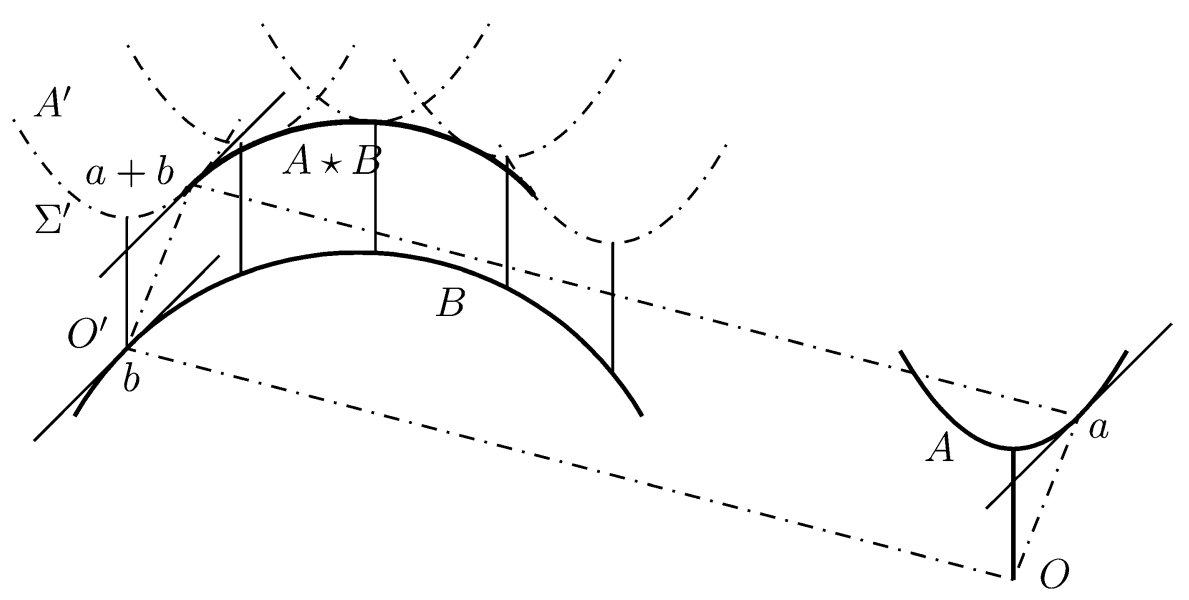

Fig. 4. Kinematic generation of convolutions in the curve case.

The convolution $A \star B$ admits the following kinematic interpretation. ${ }^{2}$ Consider the surface $A$ together with the origin $O$ as a moving system $\Sigma^{\prime}$ and let $B$ be fixed, and let $A^{\prime}$ and $O^{\prime}$ denote the different positions of $A$ and $O$. The system $\Sigma^{\prime}$ is moved translatory (without any rotational part) such that the point $O^{\prime}$ travels on the second surface $B$. The convolution $A \star B$ is generated as the envelope of $A^{\prime}$ under this two-parameter translational motion. The curve case is visualized in Fig. 4.

In particular, if the surface $A$ is a sphere with radius $d$, centered at $O$, then the convolution surface $A \star B$ becomes the (untrimmed) offset surface of $B$ at distance $d$.

\section{Parameterization of convolution surfaces}

After discussing the general case, we compute convolution surfaces of general rational surfaces and LN surfaces.

\subsection{Computation of convolution surfaces}

Consider again two surfaces $A$ and $B$, which are given by parametric representations $\mathbf{a}(u, v)$ and $\mathbf{b}(s, t)$ with parameter domains $\Omega_{A}, \Omega_{B}$. Let $\overrightarrow{\mathbf{M}}(u, v)$ and $\overrightarrow{\mathbf{N}}(s, t)$ be their normal vectors, and

$$
\mathbf{M}_{0}(u, v)=\frac{\mathbf{M}(u, v)}{\|\mathbf{M}(u, v)\|}, \quad \mathbf{N}_{0}(s, t)=\frac{\mathbf{N}(s, t)}{\|\mathbf{N}(s, t)\|}
$$

the corresponding unit normal vectors. In order to find the convolution surface, we have to construct a reparameterization

$$
\phi: \Omega_{B}^{*} \rightarrow \Omega_{A}:(s, t) \mapsto(u(s, t), v(s, t))
$$

which is defined for a certain subset $\Omega_{B}^{*} \subseteq \Omega_{B}$, such that the normal vectors $\overrightarrow{\mathbf{M}}_{0}(u(s, t), v(s, t))$ and $\overrightarrow{\mathbf{N}}_{0}(s, t)$ are parallel.

\footnotetext{
$\overline{2}$ A slightly different kinematic generation of $A \star B$ has been discussed by Mühlthaler and Pottmann (2003).
} 
The set $\Omega_{B}^{*}$ should be chosen as the maximal subset of $\Omega_{B}$, such either the Gaussian image $\overrightarrow{\mathbf{N}}_{0}\left(\Omega_{B}^{*}\right)$ or the reflected Gaussian image $-\overrightarrow{\mathbf{N}}_{0}\left(\Omega_{B}^{*}\right)$ of $B$ is contained in the Gaussian image $\overrightarrow{\mathbf{M}}_{0}\left(\Omega_{A}\right)$ of $A$. In addition, we assume that the unit normals of the first surface $\overrightarrow{\mathbf{M}}_{0}(u, v)$ define a bijective mapping $\Omega_{A} \rightarrow$ $\overrightarrow{\mathbf{M}}_{0}\left(\Omega_{A}\right)$, and $\overrightarrow{\mathbf{M}}_{0}\left(\Omega_{A}\right)$ is contained in an open hemisphere of the unit sphere. ${ }^{3}$ Under these assumptions, the reparameterization exists and it is unique.

Then,

$$
\mathbf{c}=\mathbf{a}(u(s, t), v(s, t))+\mathbf{b}(s, t)
$$

is a parametric representation of the convolution surface of $A^{*}=\mathbf{a}\left(\phi\left(\Omega_{B}^{*}\right)\right)$ and $B^{*}=\mathbf{b}\left(\Omega_{B}^{*}\right)$. For general rational surfaces $A$ and $B$, this reparameterization cannot be written down explicitly.

\subsection{Convolution of LN surfaces and rational surfaces}

In this section we want to investigate parameterizations of the convolution $A \star B$ of an $\mathrm{LN}$-surface $A$ and a rational surface $B$. We may assume that the coordinate system has been chosen such that the $\mathrm{LN}$-surface $A$ is given by a parameterization

$$
\mathbf{a}(u, v)=\left(-f_{u},-f_{v},-f+u f_{u}+v f_{v}\right) .
$$

As observed earlier in Section 2, the normal vector $\overrightarrow{\mathbf{M}}$ of $A$ is proportional to $\overrightarrow{\mathbf{M}}(u, v)=(u, v, 1)$ at regular points (which are characterized by $f_{u u} f_{v v}-f_{u v} \neq 0$ ). In this case, the unit normals $\overrightarrow{\mathbf{M}}_{0}(u, v)$ are contained in the upper hemisphere.

For the sake of simplicity, we choose $\Omega_{A}=\mathbb{R}^{2}$ throughout this section. The second surface $B$ is assumed to admit a smooth local parameterization

$$
\mathbf{b}:(s, t) \in \Omega_{B} \subset \mathbb{R}^{2} \rightarrow \mathbb{R}^{3} .
$$

Two points $\mathbf{a} \in A$ and $\mathbf{b} \in B$ correspond to each other, if the normal vectors $\overrightarrow{\mathbf{M}}$ and $\overrightarrow{\mathbf{N}}$ at $\mathbf{a}$ and $\mathbf{b}$ are linearly dependent,

$$
\overrightarrow{\mathbf{M}}(\mathbf{a})=\lambda \overrightarrow{\mathbf{N}}(\mathbf{b}), \quad \lambda \neq 0 .
$$

Then, $\mathbf{a}+\mathbf{b}$ is a point of the convolution surface $C=A \star B$.

Using the normal vector $\overrightarrow{\mathbf{N}}(s, t)=\left(n_{1}(s, t), n_{2}(s, t), n_{3}(s, t)\right)$ of $B$, the condition (25) can be rewritten as

$$
(u, v, 1)=\lambda\left(n_{1}, n_{2}, n_{3}\right)(s, t),
$$

which implies

$$
u(s, t)=\frac{n_{1}(s, t)}{n_{3}(s, t)} \quad \text { and } \quad v(s, t)=\frac{n_{2}(s, t)}{n_{3}(s, t)}
$$

provided that $n_{3}(s, t) \neq 0$. The latter condition is satisfied, since the (possibly reflected) Gaussian image $\overrightarrow{\mathbf{N}}_{0}\left(\Omega_{B}^{*}\right)$ is assumed to be contained in $\overrightarrow{\mathbf{M}}_{0}\left(\Omega_{A}\right)$.

\footnotetext{
$\overline{3}$ This is the case if and only if there exists a vector $\overrightarrow{\mathbf{z}}_{0}$, such that $\overrightarrow{\mathbf{N}}_{0}(u, v) \cdot \overrightarrow{\mathbf{z}}_{0}>0$ holds for all $(u, v) \in \Omega_{A}$.
} 


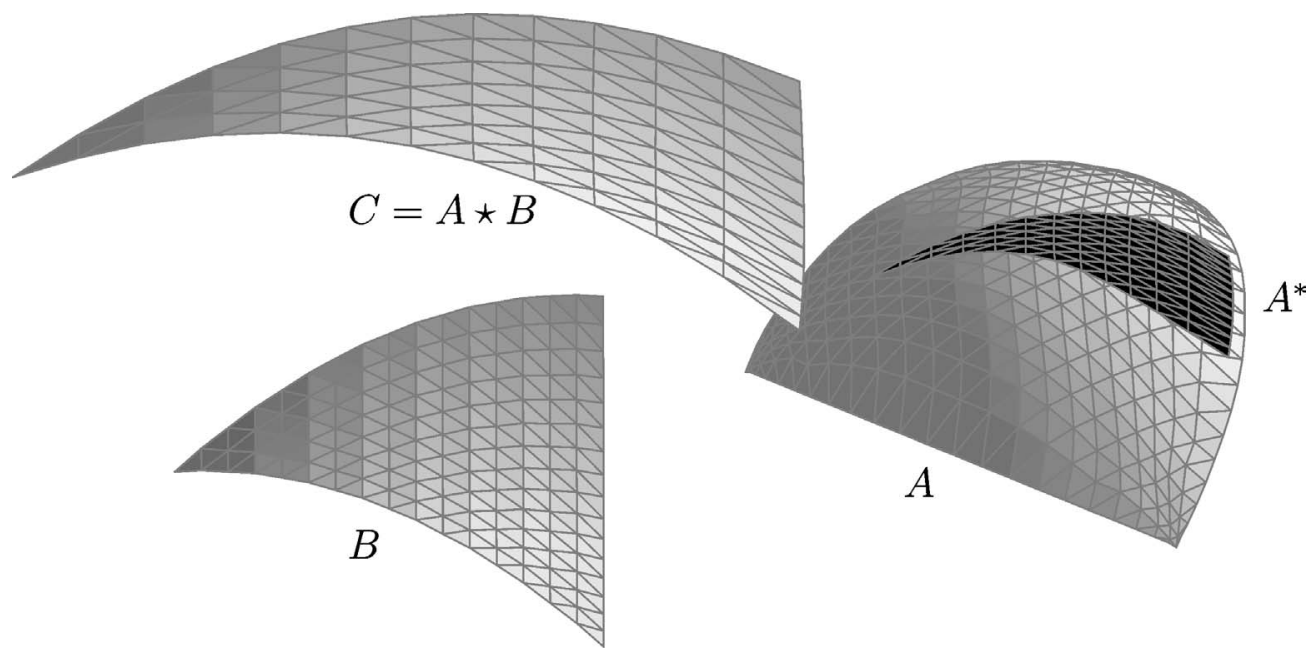

Fig. 5. Convolution surface $C$ of a triangular patch of an $\mathrm{LN}$ surface of degree $6(A)$ and a quadratic triangular patch $B$. The convolution surface is rational surface of degree 12 . Only the points contained in $A^{*}$ contribute to $C$.

The parametric representation $\mathbf{c}(s, t)$ of the convolution $C=A \star B$ is now obtained by applying the reparameterization (27) to $A$ and evaluating the sum

$$
\mathbf{c}(s, t)=\mathbf{a}\left(\frac{n_{1}(s, t)}{n_{3}(s, t)}, \frac{n_{2}(s, t)}{n_{3}(s, t)}\right)+\mathbf{b}(s, t) .
$$

If $B$ is a rational surface, the reparameterization $\phi:(s, t) \rightarrow(u, v)$ is a rational mapping and the convolution $C=A \star B$ is a rational surface.

Theorem 9. The convolution surface $A \star B$ of an $L N$-surface $A$ and a parameterized surface $B$ has an explicit parametric representation. If $A$ and $B$ are rational surfaces, their convolution $A \star B$ is rational, too.

An example is shown in Fig. 5, where we visualize the convolution surface of a quadratic triangular patch with an LN surface of degree 6.

Remark 10. The reparameterization $\phi$ is regular if and only if the determinant of the Jacobian $J \phi$ does not vanish. This determinant evaluates to

$$
\operatorname{det}(J \phi)=\frac{1}{n_{3}^{3}} \operatorname{det}\left(\overrightarrow{\mathbf{N}}, \overrightarrow{\mathbf{N}}_{s}, \overrightarrow{\mathbf{N}}_{t}\right)
$$

After some computations one arrives at

$$
\operatorname{det}(J \phi)=\frac{1}{n_{3}^{3}} \operatorname{det}\left(\overrightarrow{\mathbf{N}}, \overrightarrow{\mathbf{N}}_{s}, \overrightarrow{\mathbf{N}}_{t}\right)=\frac{1}{n_{3}^{3}} \operatorname{det}\left(G_{b}\right)^{2} k_{b},
$$

where $G_{b}$ is the first fundamental form of $B$, and $k_{b}$ its Gaussian curvature. We mention two special cases which correspond to a singular Jacobian (28) of the reparameterization $\phi$ : 
- If $B$ is a plane, the unit normal vector $\overrightarrow{\mathbf{N}}_{0}$ does not depend on $s, t$, but it is constant. Since (27) gives a single point $(u, v)$, there is a single point $\mathbf{a}_{0}$ on $A$ which corresponds to all points of $B$. Thus, $A \star B$ is a plane translated by the fixed vector $\mathbf{a}_{0}$.

- If $B$ is a developable surface (i.e., its Gaussian curvature vanishes), $\phi$ maps the domain $\Omega_{B} \subset \mathbb{R}^{2}$ into a curve in the $u v$-plane. Thus, there is in general only a curve $\mathbf{a}(\tau) \in A$ which contributes to the construction of $A \star B$. Clearly, the convolution surface is again a developable surface.

Remark 11. Points of $B$ with $n_{3}(s, t)=0$ have no corresponding point on the LN-surface $A$. If there is one point with this property then, in general, there exists even a curve $c \in B$ with $n_{3}=0$ along $c$. The curve $c$ is a shadow boundary of $B$ with respect to an illumination parallel to the $z$-axis. In this case the convolution $A \star B$ consists of non-connected parts.

\section{Conclusion}

As the main result of this paper, we identified a class of free form surfaces which have rational convolution surfaces with general rational surfaces. To our knowledge, this is the first result on rational convolution surfaces of surfaces which are capable of modeling general free-form geometries. This result may serve as the starting point for research on computing Minkowski sums of general free-form objects. While the case of two convex objects should be relatively simple, the computation of the Minkowski sum of general objects will need robust methods for detecting and trimming the inner branches of the convolution surfaces, which do not contribute to the boundary of the Minkowski sum. While our attention has been mainly devoted to the surface case, the computation of convolutions of LN-curves and rational curves can be treated as a simple particular case.

\section{Acknowledgements}

A major part of this work was done during a visit of M. Peternell and B. Jüttler to Siena in February 2005. The authors are indebted to P. Costantini for supporting this visit within the frame of the FIRB project, contract n. RBAU0128CL. Additionally, this research has been supported partially by the research network S92 of the Austrian Science Fund FWF. The authors would like also to thank the referees for their valuable comments, which have helped to improve the presentation of this work.

\section{References}

Bajaj, C., Kim, M.-S., 1989. Generation of configuration space obstacles: The case of a moving algebraic curve. Algorithmica 4 (2), 157-172.

Farin, G., Hoschek, J., Kim, M.-S., 2002. Handbook of Computer Aided Geometric Design. Elsevier, Amsterdam.

Farouki, R.T., 2003. Minkowski combination of complex sets—geometry, algorithms and applications. In: Lyche, T., Mazure, M.-L., Schumaker, L.L. (Eds.), Curve and Surface Design: Saint Malo, 2002. Nashboro Press, pp. 123-146.

Jüttler, B., 1998. Triangular Bézier surface patches with a linear normal vector field. In: The Mathematics of Surfaces VIII. Information Geometers, pp. 431-446.

Jüttler, B., Sampoli, M.L., 2000. Hermite interpolation by piecewise polynomial surfaces with rational offsets. Computer Aided Geometric Design 17, 361-385. 
Kaul, A., Farouki, R.T., 1995. Computing Minkowski sums of planar curves. Int. J. Computat. Geom. Appl. 5, $413-432$.

Kohler, K., Spreng, M., 1995. Fast computation of the C-space of convex 2D algebraic objects. Int J. Robotics Res. 14 (6), 590-608.

Landsmann, G., Schicho, J., Winkler, F., 2001. The parametrization of canal surfaces and the decomposition of polynomials into a sum of two squares. J. Symbolic Comput. 32 (1-2), 119-132.

Lee, I.-K., Kim, M.-S., Elber, G., 1998a. Polynomial/rational approximation of Minkowski sum boundary curves. Graphical Models 60 (2), 136-165.

Lee, I.K., Kim, M.-S., Elber, G., 1998b. The Minkowski sum of 2D curved objects. In: Proceedings of Israel-Korea Bi-National Conference on New Themes in Computerized Geometrical Modeling, Tel-Aviv University, pp. 155-164.

Li, A.-M., Simon, U., Zhao, G., 1993. Global Affine Differential Geometry of Hypersurfaces. de Gruyter, Berlin.

Lü, W., 1994. Rationality of the offsets to algebraic curves and surfaces. Appl. Math.-JCU 9B, 265-278.

Mühlthaler, H., Pottmann, H., 2003. Computing the Minkowski sum of ruled surfaces. Graphical Models 65, 369-384.

Peternell, M., Pottmann, H., 1998. A Laguerre geometric approach to rational offsets. Computer Aided Geometric Design 15, 223-249.

Peternell, M., Manhart, F., 2003. The convolution of a paraboloid and a parametrized surface. J. Geometry Graph. 7, $157-171$.

Pottmann, H., 1997. General offset surfaces. Neural Parallel Scientific Comput. 5, 55-80.

Ramkumar, G.D., 1996. An algorithm to compute the Minkowski sum outer-face of two simple polygons. In: Proc. ACM Symposium on Computational Geometry.

Sampoli, M.L., 2005. Computing the convolution and the Minkowski sum of surfaces. In: Proceedings of the Spring Conference on Computer Graphics, Comenius University, Bratislava. ACM Siggraph, in press.

Schicho, J., 1998. Rational parametrization of surfaces. J. Symbolic Comput. 26, 1-30.

Schicho, J., 2000. Proper parametrization of real tubular surfaces. J. Symbolic Comput. 30, 583-593.

Sendra, J.R., Sendra, J., 2000. Rationality analysis and direct parametrization of generalized offsets to quadrics. Appl. Algebra Engrg. Commun. Comput. 11 (2), 111-139.

Seong, J.-K., Kim, M.-S., Sugihara, K., 2002. The Minkowski sum of two simple surfaces generated by slope-monotone closed curves. In: Proceedings of Geometric Modeling and Processing, 2002, Japan, pp. 33-42. 